splitting of a nucleus within a given electronic shell leads to a loosening of the latter, one finds: the molar refractions of $\mathrm{He}$ and $\mathrm{H}_{2}$ are $0.5 \mathrm{cc}$ and $2.0 \mathrm{cc}$; those of $\mathrm{Ne}$ and $\mathrm{N}_{2}$ are $1.0 \mathrm{cc}$ and $4.0 \mathrm{cc}$, respectively.

This loosening of the electronic system is accompanied by a strengthening of the external field of the molecule; with respect to the boiling point, $\mathrm{H}_{2}$ is nearer to $\mathrm{Ne}$ than to $\mathrm{He}$, and $\mathrm{N}_{2}$ is nearer to $\mathrm{Kr}$ than to $\mathrm{Ne}$.

The significance of the ten electron shell is also shown in a maximum of binding strength (maximum in force constant, minimum in distance), e.g., in the series $\mathrm{C}_{2}, \mathrm{~N}_{2}$, $\mathrm{O}_{2}, \mathrm{~F}_{2} ; \mathrm{BeO}, \mathrm{BO}, \mathrm{CO}, \mathrm{NO}, \mathrm{O}_{2} ; \mathrm{C}_{2}, \mathrm{C}_{2} \mathrm{H}_{2}, \mathrm{C}_{2} \mathrm{H}_{4}, \mathrm{C}_{2} \mathrm{H}_{6}$.

The above facts and the quantum formula of the neon atom $1 s^{2} 2 s^{2} 2 p^{6}$, lead one to sharpen Langmuir's picture of $\mathrm{N}_{2}$ by allocating the principal quantum number $n=1$ to a group of 2 electrons and $n=2$ to a group of eight electrons. It has been emphasized ${ }^{1}$ that the principal quantum number of the electrons nearest to the bonded cores can be expected to depend on the spacial extension of the electronic system of the latter. The fact that $\mathrm{N}^{5+}$ is much smaller than $\mathrm{Li}^{+}$and the internuclear distance in $\mathrm{N}_{2}$ $(r=1.09 \mathrm{~A})$ is also smaller than in $\mathrm{Li}_{2}(r=2.67 \mathrm{~A}, n=2)$ gives additional support to the quantum formula of $\mathrm{N}_{2}$ : $K K ; 1^{22}$. A subdivision of the eight electron shell has to be based on spectroscopic considerations. ${ }^{2 b}$

While each of the two pairs of $K$ electrons of $N_{2}$ is quantized with respect to one of the two nuclei $\mathrm{N}^{7+}$, the third pair with $n=1$ and the 8 electrons with $n=2$ are quantized with respect to the field of both $\mathrm{N}^{5+}$. Therefore the above does not contradict the Pauli principle which in its original form applies to monatomic particles.

* The data on diatomic molecules used in this and the following two atters are from $G$. torzber (see letters are from $G$. Herzberg (see reference $2 b$ ). Some of the other dat are from L. Pauling, The Nature Of The Chemical Bond (Cornell Uni-

versity Press, Ithaca, New York, 1940), second edition.
I T. Berlin and K. Fajans, J. Chem. Phys. 10, 691 (1942).

$1 \mathrm{~T}$. Berlin and $\mathrm{K}$. Fajans, J. Chem. Phys. 10, 691 (1942).
$2 \mathrm{a}$. The methods of applying quantum theory to molecules de2 a. The methods of applying quantum theory to molecules de-
veloped by Heitler, London, Pauling, and Slater as well as that of Hund, Lennard-Jones, and Mulliken compare the molecules with atoms. b. See G. Herzberg, Molecular Spectra and Molecular Structure

${ }^{3}$ I. Langmuir, J. Am. Chem. Soc. 41, 901 (1919). See also W. Kossel, nn. d. Physik 49, 360 (1916).

4 K. Fajans and T. Berlin, Buffalo Meeting of the American Chemical Society, September, 1942. The detailed papers will be published soon. 5 E.g., the molar refraction increases in the series $\mathrm{Ne}, \mathrm{HF}, \mathrm{H}_{2} \mathrm{O}$ $\mathrm{H}_{3} \mathrm{~N}$, $\mathrm{H}_{4} \mathrm{C}$ from 1.0 to $6.5 \mathrm{cc}$. (See N. Bauer and K. Fajans, J. Am. $\mathrm{Ch}_{2} \mathrm{H}_{6}$ is $12.9 \mathrm{cc}$.

\section{Difficulties in the Valence Bond Theory*}

\author{
Kasimir Fajans \\ Department of Chemistry, University of Michigan, \\ Ann Arbor, Michigan \\ November 12, 1942
}

T

$\mathrm{HE}$ subdivision of the 10 valence electrons of $\mathrm{N}_{2}$ into the groups of 2 and 8 electrons (see III) is not in accord with its usual electronic formula : $\mathrm{N}::: \mathrm{N}:$ indicating a triple bond.

It can be shown that this contradiction adds to the difficulties of Kekulé's valence bond theory and its usual electronic interpretation.

In $\mathrm{N}_{2}{ }^{+}$the force constant is smaller, the internuclear distance (1.117A) is larger than in $\mathrm{N}_{2}$. Therefore, the detached tenth electron strengthened the binding of the two $\mathrm{N}^{5+}$ cores. One has to conclude that the other 9 electrons are also bonding since the ionization process eliminates the electron which is most loosely bound and there is (see III, footnote 4) a close interrelation between the strength with which the electrons are bound and that with which they bind the cores. Thus, all ten and not merely six electrons take part in the binding of the cores., ${ }^{1,2}$

The usual valence bond formula of $\mathrm{N}_{2}$ fits into the series $\mathrm{F}-\mathrm{F}, \mathrm{O}=\mathrm{O}, \mathrm{N} \equiv \mathrm{N}$ which one is inclined to extrapolate to $\mathrm{C} \equiv \mathrm{C}$. The increase in dissociation energy into atoms from $\mathrm{F}_{2}$ (65 kcal.) to $\mathrm{N}_{2}(170 \mathrm{kcal}$.) could be considered as a further support of these formulae, since the energy of the carbon-carbon bond increases in the series single, double, triple bond. ${ }^{3}$ From this point of view one would expect that the bond $\mathrm{C} \equiv \mathrm{C}$ is still stronger than $\mathrm{N} \equiv \mathrm{N}$ or $-\mathrm{C} \equiv \mathrm{C}-$. Contrary to that, the dissociation energy of $\mathrm{C}_{2}$ into atoms is $83 \mathrm{kcal} .$, i.e., of the order of magnitude of the single bond only. Moreover, $\mathrm{C}_{2}$ has a very strong external field and thus has no noble gas character. One has also to recall that the electronic formula : $\ddot{O}:: \ddot{0}:$ has already been disproved by the paramagnetism of $\mathrm{O}_{2}$ and that other formulae have been proposed for it, e.g., : $: \ddot{0}: \ddot{\mathrm{O}}:$ by G. N. Lewis. ${ }^{4}$

Thus among the valence bond formulae of the mentioned four diatomic molecules, $: \ddot{\mathrm{F}}: \ddot{\mathrm{F}}:$ is the only one which is not in disagreement with the behavior of these elementary substances.

The usual electronic formula of $\mathrm{N}_{2}$ and many others based on the idea of completion of a noble gas shell by sharing electrons also encounter the following difficulty. Only a limited number of atoms or radicals can assume a noble gas configuration by an exothermic process: $H, F$, $\mathrm{Cl}, \mathrm{Br}, \mathrm{I}, \mathrm{OH}, \mathrm{CN}$, and perhaps some of the polyatomic radicals.

The oxygen ion $\mathrm{O}^{-}$, however, is unstable in the free gaseous state and its electronic shell has to be stabilized, e.g., by $\mathrm{H}^{+}$in $\mathrm{OH}^{-}$or by other cations (V). This is also true for $\mathrm{N}^{3-}, \mathrm{C}^{4-}$, or $\mathrm{B}^{5-}$. Thus, in a symmetrical molecule $\mathrm{N}_{2}$ one could assume a temporary polarity (II) connected with a completion of one $\mathrm{N}^{3-}$ stabilized by $\mathrm{N}^{3+}$ from the other atom. The above consideration contradicts, however, the assumption that in $\mathrm{O}_{2}, \mathrm{~N}_{2}, \mathrm{C}_{2}$ both atoms at the same time complete their octets.

The contention (see Pauling, III*) that many molecules, even the simplest, are the result of the resonance between a number of forms with different combinations of single, double, and other bond types can be considered as a strong criticism of the valence bond theory. For it means the sacrifice of the initial aim of the theory to represent one experimentally homogeneous molecular species by one formula.

The quantum formula of $\mathrm{N}_{2}, K K ; 1^{2} 2^{8}$, (see III) is free of these difficulties. It considers all ten electrons as common to both cores and resembles in this respect the method of molecular orbitals used (III, 2a) in the discussion of spectral data. There are, however, many types of substances to which neither a valence bond formula nor an 
electronic shell common to the whole molecule can be assigned. They are discussed in the following letter.

*This is communication IV on "Electronic Structure of Molecules." The other communications in the J. Chem. Phys. will be quoted by the following Roman numbers: I. K. Fajans and N. Bauer, 10, 410 (1942); II. T. Berlin and K. Fajans, 10, 691,

1 This bonding has to be considered as due to the electron density 1 This bonding has to be considered as due to the electron density present between the cores. As has been shown $(I I, 4)$ it is possible on
this basis to interrelate properties of molecules which do not differ with respect to quantization.

with respect to quantization. the interaction between atoms and not between cores and electrons, it the interaction between atoms and not between cores and electrons, it appears to be possible (III, $2 b$ ) to correlate the spectroscopic properties acted as bonding, two as antibonding, and that in the balance the bond between the atoms is due to six electrons.

${ }^{3} \mathrm{~K}$. Fajans, Ber. d. D. Chem. Ges. 53, 643 (1920); 55, 2826 (1922).

4 G. N. Lewis, Chem. Rev. 1, 231 (1924).

\section{Stabilization and Binding of Quantized Radicals*}

\author{
Kasimir Fajans \\ Department of Chemistry, University of Michigan. \\ Ann Arbor, Michigan \\ November 12, 1942
}

$I^{\mathrm{N}}$ $\mathrm{N}$ any attempt to reach a uniform point of view concerning the electronic structure of molecules, one has to consider the electric interaction and the quantization.

Using a few characteristic examples we shall combine these two factors into a principle which seems to have broad application to chemical binding.

The oxide $\mathrm{MgO}$ can be considered as ionic. However, $\mathrm{O}^{-}$in the free state is unstable with respect to $\mathrm{O}^{-}$and $e^{-}$(I) and, therefore, the principal quantum number of its eighth electron is $n=\infty$. When combined with $\mathrm{Mg}^{++}$the value of $n$ is reduced, in all probability, to $n=2$. This process requires energy and, thus, the neon state of $\mathrm{O}^{-}$has to be stabilized by the binding process.

This point of view can be applied to an electron pair. Left to itself the pair would dissociate. However, it can be stabilized by positive charges, in $\mathrm{H}^{-}, \mathrm{He}, \mathrm{Li}^{+}$, as well as in $\mathrm{H}_{2}, \mathrm{Li}_{2}$ and represents a spacial distribution of negative charge. Depending on the field strength of the positive charges, the pair can be expected to assume a larger or smaller size with unchanged principal quantum number $n$, but can also differ in quantization as has been concluded (II) for $\mathrm{H}_{2}$ and $\mathrm{Li}_{2}$.

One characteristic example of this analogy between $\mathrm{O}^{=}$ and an electron pair will be given here. The $\mathbf{P}$ atoms in $\mathrm{P}_{4} \mathrm{O}_{10}$ form a tetrahedron, 6 of the $\mathrm{O}$ atoms are situated near the middle of its 6 edges, and each of the remaining 4 is attached directly to one $\mathrm{P}$ atom. The $\mathrm{P}-\mathrm{O}$ distances ${ }^{1}$ for the 4 oxygens are shorter than those for the 6 oxygens, which can be easily understood if one derives the molecule from $\mathrm{P}^{5+}$ and $\mathrm{O}^{-}$. The one-sided polarization leads to shorter distances than a many-sided polarization. ${ }^{2}$ The structure of $\mathrm{P}_{4} \mathrm{O}_{6}$ is similar to that of $\mathrm{P}_{4} \mathrm{O}_{10}$ suggesting that the $4 \mathrm{O}^{-}$have been replaced by 4 unshared electron pairs. Finally, in $\mathrm{P}_{4}$, which also has a tetrahedral structure, the $6 \mathrm{O}^{=}$of $\mathrm{P}_{4} \mathrm{O}_{6}$ can be considered to be replaced by electron pairs, each pair shared by two $\mathrm{P}^{5+}$.

Coordination compounds are characterized by the binding of stable molecules or of radicals stabilized by the binding process. $\dagger$ The remarkable group of isoelectronic substances $\mathrm{Ni}(\mathrm{CO})_{4}, \mathrm{Co}(\mathrm{CO})_{3}(\mathrm{NO})$, and $\mathrm{Fe}(\mathrm{CO})_{2}(\mathrm{NO})_{2}$ is diamagnetic. The $\mathrm{C}-\mathrm{O}$ distance $(1.15 \pm 0.03 \mathrm{~A})^{3}$ in these compounds differs slightly from that in free $\mathrm{CO}(1.128 \mathrm{~A})$, the $\mathrm{N}-\mathrm{O}$ distance $(1.11 \pm 0.04 \mathrm{~A})$ is near that in $\mathrm{NO}^{+}$ $(1.073 \mathrm{~A})$. We suggest that the compounds contain deformed $\mathrm{CO}$ and $\mathrm{NO}^{+}$, both of which have great stability as particles with ten electrons (III). Accordingly, the metals are present in these compounds in the isoelectronic form $\mathrm{Ni}, \mathrm{Co}^{-}$, and $\mathrm{Fe}^{-\infty}$, having the electron configuration $K^{2} L^{8}(M+N)^{18}$. In the normal stable paramagnetic state of the $\mathrm{Ni}$ atom, the electrons beyond the $L$ shell are subdivided into $3 s^{2} 3 p^{6} 3 d^{8}$ and $4 s^{2}$. The transfer of the two $\mathrm{N}$ electrons into the $M$ shell would complete it and make it diamagnetic. We therefore conclude that this state of higher energy is stabilized by the energy of binding between particles with 10 and 18 electrons.

The boron hydrides can be fitted into the above scheme, e.g., $\mathrm{B}_{4} \mathrm{H}_{10}$ can be formulated as $\mathrm{BH}_{3} \cdot \mathrm{B}_{2} \mathrm{H}_{4} \cdot \mathrm{BH}_{3}$. The radical $\mathrm{B}_{2} \mathrm{H}_{4}$ is a member of the isoelectronic series $\mathrm{N}_{2}$, $\mathrm{HCN}, \mathrm{HCCH}, \mathrm{H}_{2} \mathrm{BBH}_{2}$ in which the successive splitting of the nucleus (III) leads to the loosening of the system. Similarly, $\mathrm{B}_{5} \mathrm{H}_{9}$ is $\mathrm{B}_{4} \mathrm{H}_{6} \cdot \mathrm{BH}_{3}$, the former of the two radicals having an 18 electron shell. ${ }^{4}$

The above offers examples of stabilization of radicals with various numbers of electrons, $2\left(2 e^{-}\right), 6\left(\mathrm{BH}_{s}\right), 8\left(\mathrm{O}^{-}\right)$, $10\left(\mathrm{~B}_{2} \mathrm{H}_{4}\right), 18(\mathrm{Ni})$ within ionic, covalent, and coordination compounds. In papers to follow this binding principle will be extended to many other cases.

I appreciate the valuable discussions with Professor Norman Bauer, University of New Hampshire, and $\mathrm{Mr}$. Theodore Berlin.

* This is communication $V$ on "Electronic Structure of Molecules."

See communication IV, preceding letter.
1 L. R. Maxwell, J. Opt. Soc. Am. 30, 374 (1940). As will be shown in a detailed paper, the gradation of the distances in $\mathrm{P}_{4}, \mathrm{P}_{4} \mathrm{O}_{3}, \mathrm{P}_{4} \mathrm{O}_{10}$ can be understood from the above point of view.

2 K. Fajans, J. Chem. Phys. 9, 378 (1941).

+ The binding is due to dipoles and polarizability

${ }^{\dagger} \mathrm{L}$. O. Brockway and J. S. Anderson, Trans. Faraday Soc. 33, 1233 (1937)

${ }^{4}$ The proposed structures are in agreement with the electron diffraction results of S. H. Bauer and L. Pauling, J. Am. Chem. Soc. 58, 2403 (1936). 